\title{
Ensayos para la refinacién física de la manteca de karité
}

\author{
Por M. V. Ruiz Méndez, J. Huesa Lope, J. Pereda y M. C. Dobarganes \\ Instituto de la Grasa y sus Derivados, C.S.I.C. \\ Avda. P. García Tejero, 4. 41012 - Sevilla
}

RESUMEN

Ensayos para refinación física de la manteca de karité.

Se estudian las condiciones más adecuadas en la refinación de la manteca de karité para su uso en cosmética. El objetivo es conseguir un producto decolorado, desodorizado con acidez libre inferior al $3 \%$ e insaponificable elevado, con pérdidas minimas sobre el existente en la muestra inicial.

Se han estudiado las siguientes variables: proporción de áciđó fosfórico en la fase de desgomado, cantidad de tierras decolorantes y carbón activo en la decoloración y temperatura/tiempo en la desodorización. Los resultados demuestran que puede obtenerse una grasa de buena calidad mediante refinación física cuando las muestras contienen menos de un $5 \%$ de acidez. En caso contrario es necesario una neutralización previa que debe ser parcial para disminuir al mínimo las pérdidas de compuestos del insaponificable.

PALABRAS-CLAVE: Insaponificable - Manteca de karité Refinación física. •

\section{SUMMARY}

Assays for physical refining of shea butter.

The best conditions for the refining of shea butter used in cosmetic industries are defined. The objective is to obtain a bleached, deodorized fat, with a $3 \%$ maximun free acidity and a high amount of unsaponifiable matter having minimal losses with respect to the intitial sample.

The following variables have been studied: phosphoric acid proportion in degumming, bleaching clays and active carbon percentages in bleaching, and temperature/time in the stage of deodorization.

The results demonstrate that good quality shea butter can be obtained by physical refining if the level of free fatty acid in the initial sample is lower than $5 \%$. Otherwise, a neutralization is necessary, which should be partial in order to reduce the loss of unsapónifiatble matter.

KEY-WORDS: Physical refining - Shea butter - Unsaponifiable.

\section{INTRODUCCION}

La manteca de karité, utilizada tradicionalmente en los países africanos para el cuidado de la piel y como alimento, está siendo introducida en Europa en cantidades importantes debido a sus aplicaciones en farmacología y cosmética. (1) (2).

Su uso en los sectores señalados, donde adquiẹ:re un elevado valor añadido, obliga a preparar una materia prima con unas características muy especificas de calidad que se resumen en las siguientes:

- Acidez inferior a 3\% (como tanto por ciento de ácido oleico).

- Color no superior a 4 en escala Gardner.

- Ausencia total de olores extraños.

El valor de comercialización de la manteca, por otra parte, es más elevado cuanto mayor es el contenido en fracción insaponificable.

La mayor parte de la manteca extraída tiene valores de acidez elevados (superior al $4 \%$ ), un color oscuro y un olor desagradable muy acentuado, por lo que es necesario modificar su composición para adecuarla a las especificaciones establecidas para su comercialización.

Dadas las características definidas, es obvio que la refinación constituye el proceso más adecuado de tratamiento, aunque para conseguir el producto deseado se tienen que establecer modificaciones sobre los objetivos clásicos de algunas de las etapas de la refinación de aceites y grasas comestibles.

Por ello, el objetivo de este estudio es definir las mejores condiciones de refinación de la manteca de karité para obtener un producto que mantenga su característica más apreciada (alto contenido en fracción insaponificable) y reúna las especificaciones previamente establecidas, minimizando las pérdidas.

Con este fin se han planteado experiencias para establecer en las distintas etapas del proceso, los mejores valores de las variables que hagan compatible el objetivo a alcanzar en cada fase con el mantenimiento de la fracción insaponificable en los valores iniciales.

\section{PARTE EXPERIMENTAL}

\subsection{Muestras utilizadas}

Las muestras recibidas son manteca de karité procedentes de Alto Volta, facilitadas por una empresa especializada en el campo de la cosmética. 
Se han estudiado dos muestras distintas, cuyas características más relevantes se muestran en la Tabla I (Indices generales) y en la Tabla II (Composición en ácidos grasos).

Tabla I

Características de las muestras iniciales.

\begin{tabular}{|c|c|c|}
\hline PARAYRTROS & $\mathbf{A}$ & B \\
\hline ACIDEZ (\% oleico) & 4.7 & 7.0 \\
\hline HUKGEAD $(g / 100 \mathrm{~g})$ & 0.58 & 0.20 \\
\hline IMPUREZAS $(g / 100 \mathrm{~g})$ & $<0.05$ & 0.11 \\
\hline INSAPOU IFICABLE (\%) & 5.6 & 7.6 \\
\hline ACIDOS OXIDADOS (x) & 0.17 & 0.17 \\
\hline COLOR (E.Gardner) & $9-10$ & $9-10$ \\
\hline I IRDICE DE YODO $\left(\mathrm{n}-\mathrm{g} \mathrm{I}_{z} / 100 \mathrm{~g}\right)$ & 56.4 & 58.6 \\
\hline FOSFORO (ppm) & 70 & 100 \\
\hline
\end{tabular}

Tabla II

Composición en ácidos grasos de las muestras iniciales.

\begin{tabular}{|lrr|}
\hline ACIDO & $A(\%)$ & $B(\%)$ \\
\hline PALMITICO & 3.8 & 3.7 \\
ESTEARICO & 37.5 & 38.3 \\
OLEICO & 49.7 & 50.8 \\
LIMOLEICO & 7.9 & 6.5 \\
ARAQUICO & 0.9 & 0.8 \\
\hline
\end{tabular}

\subsection{Tratamiento de las muestras}

Las muestras se han sometido a refinación física en un sistema discontinuo de laboratorio, en el que se trabaja con cantidades de muestra que permitan extrapolar los resultados obtenidos y fijar las condiciones de cada etapa del proceso en planta piloto.

\section{Depuración.}

Aproximadamente $1 \mathrm{~kg}$ de grasa se introduce en un vaso de precipitado de $2 \mathrm{I}$. Se funde y mediante un agitador de paletas, se agita a una velocidad de $120 \mathrm{rpm}$. Se añade $\mathrm{H}_{3} \mathrm{PO}_{4} 7 \mathrm{M}$ (calidad alimentaria) en distintas proporciones para seleccionar la más adecuada, (ver Tabla III). Se mantiene la agitación, aumentando la temperatura hasta $70^{\circ} \mathrm{C}$ y durante un tiempo máximo de 20 minutos. Pasado este tiempo se detiene la agitación y se separan los fosfátidos que hayan precipitado o floculado por decantación.

Tabla III

Cuantificación del insaponificable (\%).

\begin{tabular}{|c|c|c|}
\hline & $\begin{array}{l}\text { METODO } \\
\text { HORMA UEE }\end{array}$ & $\begin{array}{c}\text { EXTRACTOR } \\
\text { LIQUIDO - LIQUIDO }\end{array}$ \\
\hline MAITECA A CRUDA & $\begin{array}{l}\vec{x}=4.4 \\
s_{x}=0.3\end{array}$ & $\begin{array}{l}\bar{x}=5.6 \\
s_{x}=0.1\end{array}$ \\
\hline MAITECA B CRUDA & $\begin{array}{l}x=6.1 \\
s_{2}=0.4\end{array}$ & $\begin{array}{l}x=7.6 \\
s x=0.1\end{array}$ \\
\hline
\end{tabular}

A continuación se lava la grasa con un $15 \%$ en peso de agua destilada a $70^{\circ} \mathrm{C}$, hasta que se eliminen los jabones $y$, finalmente, se seca.

\section{Decoloración.}

El aceite, lavado y seco, se calienta hasta la temperatura de trabajo en atmósfera inerte. La muestra se somete a agitación y se añade la tierra decolorante (Tierra de Gador tipo C) y el carbón activo (N2G) en distintas proporciones (ver Tabla III). Se mantiene a la temperatura indicada bajo agitación durante $15 \mathrm{~min}$. $y$, a continuación, se filtra.

\section{Desodorización neutralizante.}

El sistema utilizado en el laboratorio se muestra en la figura.

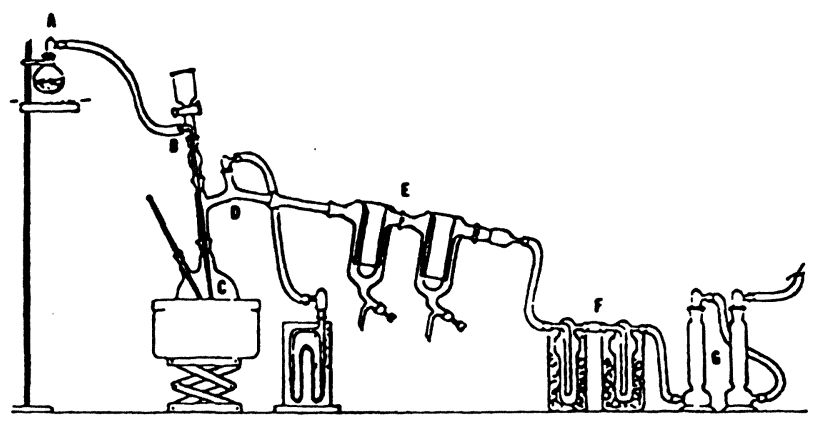

Figura

Desodorizador de laboratorio.

La grasa decolorada se introduce en el matraz (C) del esquema adjunto, el cual se calienta mediante una manta calefactora semiesférica con un termorregulador. La fuente de vapor de agua para la desodorización es un matraz de $100 \mathrm{ml}$. El tubo (B) dirige el vapor hacia el fondo del matraz desodorizador $y$ 
lleva una válvula para regular mejor el flujo de entrada.

El tubo de salida (D) dirige los vapores hacia una serie de trampas de condensación $(E, F, G)$ las cuales recogen el material condensable y evitan que el vapor de agua pase a la bomba de vacío, que se une al sistema por la trampa $G$.

Una vez transcurrido el tiempo establecido a la temperatura de operación, (ver Tabla IV), se enfría la grasa y el vacío se reemplaza por atmósfera de nitrógeno para evitar la oxidación.

\section{Tabla IV}

Variables utilizadas en los ensayos de refinación física de la manteca de karité.

\begin{tabular}{|c|c|c|c|c|c|c|}
\hline Depuración & $\mathrm{H}_{3} \mathrm{PO}_{4} 7 \mathrm{X}(\%$ peso $) 0$ & .4 & & & & 0.8 \\
\hline \multirow[t]{2}{*}{ Decoloración } & Tlerra (z) & 1 & 1 & 2 & & 2 \\
\hline & Carbón (x) & 0 & 0.5 & 0 & & 0.5 \\
\hline \multirow[t]{2}{*}{ Desodorizac1ón } & Temperatura $\left({ }^{\circ} \mathrm{C}\right)$ & 220 & 220 & 250 & 250 & 250 \\
\hline & Tlempo (b) & 2 & 3 & 3 & 2 & 1 \\
\hline
\end{tabular}

\subsection{Metodología analítica}

Determinación de la fracción insaponificable. En un matraz de destilación de $250 \mathrm{ml}$ se pesan, con precisión de $0.001 \mathrm{~g}, 5 \mathrm{~g}$ de muestra y se añaden 50 $\mathrm{ml}$ de solución alcohólica de $\mathrm{KOH} 2 \mathrm{~N}$; se coloca un refrigerante y se hierve a reflujo durante 1 hora, a fin de que tenga lugar la reacción de saponificación. Una vez fría, se vierte la solución jabonosa en un extractor líquido-líquido, lavándose el matraz con $100 \mathrm{ml}$ de agua destilada utilizada en varias porciones. La extracción de la fracción insaponificable se lleva a cabo con hexano durante 10 horas en dicho extractor.

El extracto disuelto en hexano se lava con porciones de $50 \mathrm{ml}$ de etanol-agua al $50 \%$ hasta que el líquido del último lavado no dé coloración rosa al adicionarle dos o tres gotas de solución indicadora de fenolftaleína.

Se pasa la disolución de hexano a un matraz tarado; se elimina el disolvente por destilación y se seca la muestra en estufa de vacío a $40-50^{\circ} \mathrm{C}$, hasta que la pérdida de peso entre dos pesadas consecutivas sea inferior al $0,1 \%$.

Para la determinación de la acidez, (3), humedad y materias volátiles, (4), impurezas (en hexano), (5), ácidos oxidados, (6), índice de yodo, (7), determinación del fósforo, (8), composición en ácidos grasos (9), se han seguido las correspondientes normas UNE. La determinación de color se realizó mediante la Escala Gardner, (10).

\section{RESULTADOS Y DISCUSION}

Las tablas I y II resumen las características generales de las muestras de partida, que se encuadran dentro de los rangos encontrados en la bibliografía para este tipo de manteca (1).

A pesar de sus características físicas, color rojizo y olor acentuado, las muestras no presentan un contenido en ácidos oxidados, humedad e impurezas muy elevados, y podemos considerarlas poco alteradas a efectos de refinación. Como puede observarse, la acidez de ambas mantecas es elevada, e incluso, la diferencia entre las muestras es suficientemente alta como para esperar un distinto comportamiento en la fase de desacidificación.

El índice de yodo implica que es una grasa con un contenido elevado en ácidos saturados, lo que está de acuerdo con la composición en ácidos grasos, que se muestra en la Tabla II.

En cuanto a la fracción insaponificable, las muestras poseen un contenido mucho más elevado que el resto de las grasas comestibles, aunque son valores normales en el caso de la grasa de karité. Dada la importancia del insaponificable para las aplicaciones a que se destina esta grasa, su mantenimiento es prioritario y servirá como criterio de selección de las condiciones a establecer en las distintas fases del proceso. Para su determinación, en un principio se siguió el método del éter de petróleo propuesto en la norma UNE (11). Sin embargo, debido a la formación de emulsiones muy estables, difíciles de romper, se obtenía baja exactitud y reproducibilidad de los resultados, como muestra la Tabla III. Por tanto, se procedió a una modificación de la técnica operando de la forma anteriormente expuesta.

En función de las características generales se selecciona el procedimiento de refinación física. Este sistema de refinación es aconsejable para aquellas grasas cuyo contenido en fosfátidos sean bajos y con una acidez no muy elevada.

Aunque el contenido en ácidos grasos libres de las muestras utilizadas en este estudio es muy elevado como para poder ser desacidificadas mediante destilación, hay que tener en cuenta que el objetivo es conseguir una acidez inferior al $3 \% \mathrm{y}$, por tanto, la acidez diferencial a eliminar es de $1,7 \%$ y $4 \%$ para las muestras $A$ y $B$, respectivamente. Ello permitía pensar que la selección era adecuada puesto que evitaba dos inconvenientes: las pérdidas de aceite neutro que conlleva la formación de pastas en la refinación química y que elevan los costes de producción, (12), y la posible modificación en la cantidad y composición del insaponificable y de la fracción de saponificables no glicéridos. 
Debido a la elevada cantidad de variables que es posible combinar en las distintas fases del proceso de refinación, se decidió un sistema de selección de variables por etapas. En cada una de las fases estudiadas se selecciona el valor de la variable que contribuye de manera más eficaz no sólo a conseguir el objetivo de la fase, sino a evitar la pérdida de insaponificable.

Los ensayos realizados para cada muestra de grasa se recogen en la Tabla IV. Además, ha sido necesario fijar otras condiciones en algunas etapas, que se comentan posteriormente.

En la Tabla $V$ se resumen los resultados obtenidos para la etapa de depuración, donde puede comprobarse que en todos los casos se alcanza el nivel de fósforo establecido. Aunque esta etapa no sería en principio necesaria dados los objetivos, es, sin embargo, imprescindible para mejor desarrollo de las restantes fases del proceso. (13) (14).

\section{Tabla V}

Fase de Depuración. Determinaciones analíticas de las muestras obtenidas utilizando distintas proporciones en peso de agente de depuración.

\begin{tabular}{|c|c|c|c|c|}
\hline $\mathrm{H} \rightarrow \mathrm{PO}_{4} 7 \mathrm{X}$ ( $\psi$ en peso) & $0^{*}$ & 0.4 & 0.6 & 0.8 \\
\hline \multicolumn{5}{|l|}{ VUESTRA A } \\
\hline FOSFORO (ppm) & 70 & $<6$ & $<6$ & $<6$ \\
\hline COLOR (E.Gardner) & $9-10$ & $7-8$ & $6-7$ & $10-11$ \\
\hline ACIDEZ (Koleico) & 4.7 & 4.7 & 4.7 & 4.9 \\
\hline $\begin{array}{l}\text { IUSAPOMIFICABLE (Z) } \\
\text { WUESTRA B }\end{array}$ & 5.6 & 5.5 & 5.6 & 4.8 \\
\hline FOSFORO (ppm) & 100 & $<6$ & $<6$ & $<6$ \\
\hline COLOR (B. läardner) & $9-10$ & $7-8$ & $7-8$ & $10-11$ \\
\hline ACIDEZ (\%oleico) & 7.0 & 7.1 & 7.1 & 7.3 \\
\hline IXSAPOIIFICABLE ( $(x)$ & 7.6 & 7.6 & 7.7 & 7.0 \\
\hline
\end{tabular}

- Muestra In1cial.

Para ambas muestras se aprecia un comportamiento similar, aunque en el caso de la muestra $A$ se obtiene una disminución significativa del color cuando se utiliza una proporción del $0,6 \%$ en peso de ácido fosfórico $7 \mathrm{M}$.

La pérdida de insaponificable para el $0,4 \%$ y $0,6 \%$ no es apreciable $y$ en cuanto a la acidez, se advierte un ligero aumento con la proporción de ácido añadido, a pesar de que tras los lavados no quede acidez mineral en el medio.

A la vista de estos resultados, se prosigue la refinación física fijando como proporción un $0,6 \%$ de ácido fosfórico $7 \mathrm{M}$, siguiendo como método operativo el procedimiento descrito anteriormente.

La decoloración se realiza en atmósfera de nitrógeno, con un agitador magnético a $700 \mathrm{rpm}$.
Como es bien conocido, la actividad de las tierras decolorantes aumenta con la temperatura hasta un máximo, a partir del cual comienza a decrecer su actividad de forma lenta, dependiendo del tipo de aceite. (15). Pruebas iniciales comparativas realizadas a distintas temperaturas, demuestran que no se obtienen mejoras acusadas de color por encima de $80^{\circ} \mathrm{C}$, temperatura seleccionada para el resto de los ensayos. El tiempo a la temperatura de operación no afecta a la grasa de manera tan crítica como la temperatura. De forma general, la eliminación de color aumenta con el tiempo, hasta un punto en el que se consigue la estabilización del mismo. La mayoría de los refinadores han establecido en 20 minutos el tiempo máximo práctico a la temperatura de trabajo. (15). En este proceso se ha mantenido un tiempo de decoloración de 15 minutos, suficiente para nuestros objetivos.

La tabla VI muestra los resultados obtenidos en las condiciones de agitación, temperatura y tiempo definidos. Dado el interés en evitar pérdidas en la fracción insaponificable, se han seleccionado proporciones bajas de tierras decolorantes ( 1 y $2 \%$ ) ya que está bien establecido que la pérdida de compuestos de la citada fracción aumenta con la cantidad de tierra decolorante utilizada. (16).

\section{Tabla VI}

Fase de decoloración. Determinaciones analíticas de las muestras obtenidas utilizando distintas proporciones de agentes decolorantes. Tiempo de operación: 15 minutos. Temperatura: $80^{\circ} \mathrm{C}$.

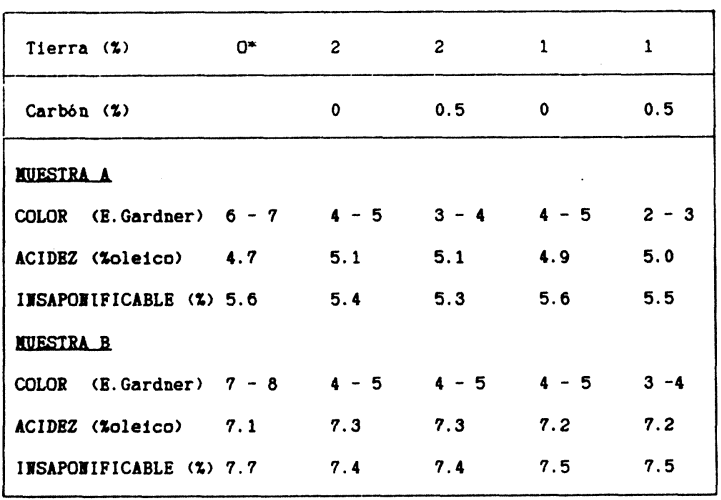

- Muestras depuradas.

Por otra parte, dadas las características de las muestras y la especificidad del carbón activo para la eliminación de las tonalidades rojas, se ha analizado igualmente la eficacia de la etapa en ausencia y en presencia de un $0,5 \%$ de carbón activo.

Los ensayos con $1 \%$ y $2 \%$ de Tierra de Gador Tipo C, como se observa en la Tabla VI, indican que las diferencias de color no son apreciables, aunque 
la acidez tiende a aumentar y el insaponificable a disminuir cuando se utiliza una proporción de tierra del $2 \%$. Esta tendencia durante la decoloración debido a la acción de las tierras, es un hecho ya indicado por diversos autores (14-16), y puede ser debido a una ligera hidrólisis ácida.

En cuanto a la adición de carbón activo, su presencia origina una mejora del color más acusada para la muestra $A$ que para la $B$, e incluso más efectiva con una menor proporción de las tierras decolorantes, aumentando la acidez de forma mínima y siendo la pérdida de insaponificable inapreciable.

Teniendo en cuenta los resultados obtenidos, se continúa la refinación tomando como parámetros de decoloración los siguientes: $1 \%$ de Tierra $+0,5 \%$ de Carbón Activo, Ta $80^{\circ} \mathrm{C}$, t $15 \mathrm{~min}$.

La Tabla VII muestra los resultados obtenidos en la etapa de desodorización neutralizante.

\section{Tabla VII}

Fase de Desodorización-neutralizante. Determinaciones analíticas de las muestras que se han obtenido utilizando distintas temperaturas y tiempos de operación.

\begin{tabular}{|c|c|c|c|c|c|c|}
\hline Temperatura $\left({ }^{\circ} \mathrm{C}\right)$ & $0^{*}$ & 220 & 220 & 250 & 250 & 250 \\
\hline Tiempo (b) & & 2 & 3 & 3 & 2 & 1 \\
\hline \multicolumn{7}{|l|}{ MANTECA A } \\
\hline COLOR (E. Gardner) & $2-3$ & $2-3$ & $2-3$ & $2-3$ & $2-3$ & $2-3$ \\
\hline ACIDEZ (\%oleico) & 5.0 & 4.2 & 4.0 & 2.2 & 2.8 & 3.6 \\
\hline IHSAPOI IFCABLE (\%) & 5.5 & 5.5 & 5.6 & 6.0 & 5.8 & 5.6 \\
\hline ZAITES:A B & & & & & & \\
\hline COLOR (E. Gardner) & $3-4$ & - & $3-4$ & $3-4$ & - & - \\
\hline ACIDEZ (zole1co) & 7.2 & - & 6.4 & 4.6 & - & - \\
\hline I HSAPOHIF ICABLE $(z)$ & 7.5 & - & 7.6 & 7.8 & - & - \\
\hline
\end{tabular}

- Muestras decoloradas.

Como es bien conocido, los parámetros más importantes a definir en esta etapa son la temperatura y el tiempo de operación, que afectan a las características organolépticas de la grasa y a su estabilidad frente a la oxidación. (17). El uso de temperaturas superiores a $270^{\circ} \mathrm{C}$, aún con cortos períodos de tratamiento, dan como resultado grasas de estabilidad muy baja, más oscuras y con mayor cantidad de ácidos grasos libres. (18). Estos cambios se deben tanto a reacciones termolíticas que originan ácidos grasos libres, como al desarrollo de las reacciones de polimerización y ciclación que incrementan la viscosidad de la grasa y el contenido en ácidos grasos isómeros. (19-21).

Respecto al contenido en materia insaponificable, cuyo mantenimiento se considera prioritario, se ha encontrado una pérdida progresiva con el incremento de tiempo y de la temperatura de trabajo. Las pérdidas más significativas se dan en esteroles y en tocoferoles, en el caso de los aceites y grasas comestibles de mayor producción. (22) (23).

Si se consideran conjuntamente las ideas anteriores puede explicarse fácilmente la selección de las variables utilizadas en esta fase del estudio:

1.-Teniendo en cuenta la presión de trabajo (5 Torr) y que los ácidos grasos libres que tienen que ser destilados son mayoritariamente el esteárico y el oleico, la mínima temperatura se encuentra alrededor de $220^{\circ} \mathrm{C}$. (24).

2.-Dada la elevada temperatura mínima, se limita el tiempo de operación a $3 \mathrm{~h}$.

3.-La temperatura máxima seleccionada es de $250^{\circ} \mathrm{C}$.

Se realizaron los ensayos especificados en la Tabla IV, comenzando, lógicamente, por la manteca de menor acidez, a temperaturas de $220^{\circ} \mathrm{C}$ y con $2 \mathrm{~h}$ de tratamiento. En estas condiciones, en las cuales sería teóricamente posible la desacidificación de la manteca, no se consiguió el objetivo deseado con la muestra $A$, que es la de menor acidez. No hay, sin embargo, variación en el color, ni en el contenido en insaponificables, obteniéndose, la desodorización completa.

Por tanto se procede a aumentar el tiempo de residencia hasta $3 \mathrm{~h}$, manteniendo la temperatura de operación. En este caso, la desodorización se realizó en ambas mantecas para comprobar si la mínima temperatura era suficiente para obtener una grasa bien desodorizada. Como puede observarse, la acidez baja pero no hasta los límites requeridos. Para las dos muestras se consigue, sin embargo, una completa desodorización.

Puesto que aumentar el tiempo de residencia, según los trabajos anteriormente citados (17) (25), resulta poco conveniente para la estabilidad de la grasa y los resultados indicaban que era necesario un período mucho más prolongado, se consideró más aconsejable un aumento en la temperatura.

Se estableció la máxima temperatura de operación $\left(250^{\circ} \mathrm{C}\right)$, con un máximo tiempo de tratamiento. Como muestra la Tabla VII, para la muestra $A$, se consigue una disminución de la acidez hasta 2,2\%, por debajo del nivel exigido. En el caso de la muestra B, la disminución de acidez es bastante apreciable pero continúa por encima del límite. Por ello no se prosiguen los ensayos de refinación física con esta muestra.

Con respecto a la muestra $A$, no hay variación en el color, pero sí un ligero aumento del contenido en insaponificables. Esto puede ser debido a que, al haber una pérdida en ácidos grasos, la proporción de materia insaponificable en el total de la muestra resulte algo más elevada. 
También cabría la posibilidad de que el aumento se debiera a un incremento en la fracción de hidrocarburos como consecuencia de la pirólisis de glicéridos, como apunta Jaward (23), pero es improbable ya que no se han sobrepasado los límites de temperaturas recomendados $y$, además, la grasa tiene un contenido bajo en ácidos grasos poliinsaturados, que son los principales afectados por la temperatura. (26).

Ya que la acidez está muy por debajo del nivel exigido, se realizaron ensayos a la temperatura de $250^{\circ} \mathrm{C}$, disminuyendo el tiempo de tratamiento. Como puede observarse en la tabla, una hora de tratamiento es insuficiente para lograr el objetivo propuesto. Los resultados obtenidos con $2 \mathrm{~h}$ de tratamiento son, sin embargo, bastante satisfactorios, ya que la acidez es muy próxima a $3 \%$ y no se aprecian variaciones en el color, manteniéndose la proporción de insaponificables. En todos los ensayos realizados se consigue una manteca muy aceptable desde el punto de vista organoléptico.

En conclusión, es posible aplicar la destilaciónneutralización parcial a una manteca decolorada de karité, cuyo contenido en ácidos grasos libres sea inferior a $5 \%$ (referido al ácido oleico), estableciendo como parámetros de operación $250^{\circ} \mathrm{C}$ durante $2 \mathrm{~h}$, como mínimo, a una presión de 5 Torr. Los resultados obtenidos, por otra parte, son de interés para decidir las condiciones de temperaturas y tiempo a utilizar en otras muestras, o la necesidad de una neutralización parcial, dependiendo de su grado de acidez.

\section{CONCLUSIONES}

1. - Desde el punto de vista analítico, es necesaria la modificación de la técnica de determinación de fracción insaponificable para este tipo de grasas, ya que la utilizada para los aceites comestibles no resulta exacta ni reproducible.

2..$^{\text {E }}$ El proceso de refinación a aplicar depende de la diferencia entre el grado de acidez de la manteca cruda y el valor final que se desee obtener.

a) Se comprueba para mantecas de karité con contenidos en acidez libre inferiores al 5\%, expresados como ácido oleico, es posible utilizar una refinación física. El proceso es más idóneo ya que de esta forma disminuyen las pérdidas de grasa y se obtiene un mayor contenido en fracción insaponificable.

b) Si la manteca bruta tiene acidez superior al $5 \%$, referido al ácido oleico, resulta muy difícil la refinación física, lo que obligaría a realizar el proceso clásico de refinación.

\section{AGRADECIMIENTO}

A D. Manuel Serrano García, Ayudante de Investigación del C.S.I.C., por su inestimable colaboración.

\section{BIBLIOGRAFIA}

1. Mensier, P.-"Dictionnaire des huiles végétaux". Encyclopédie Biologique. Tomo LII. Ed. Paul Lechevalier, Paris (1957), pág. 108-110.

2. Proserpio, G. y col.--Dal terzo mondo un nuovo lipide d'interesse cosmetico, il burro di karité". Prodotto Chim. 16 (1975) 281 284.

3. Norma UNE 55-011.-Determinación de la acidez.

4. Norma UNE 55-020.-Determinación de la humedad y materias volátiles.

5. Norma UNE 55-002.-Determinación de impurezas.

6. Norma UNE 55-008.-Determinación de ácidos oxidados.

7. Norma UNE 55-013.-Indice de yodo.

8. Norma UNE 55-108.-Determinación del contenido en fósforo.

9. Norma UNE 55-037.-Determinación de ácidos grasos por cromatografia gaseosa.

10. Norma A.O.C.S. Ja 9-87.-Determinación del color. Escala Gardner.

11. Norma UNE 55-004.-Determinación del insaponificable.

12. Tandy, D.C., y Mcpherson, W.J.-“Physical refining of edible oil".-J. Am. Oil Chemists' Soc. 61 (1984) 1254-1258.

13. Carr, R.-"Refining and degumming systems for edible fats and oils".-J. Am. Oil Chemists' Soc. 55 (1978) 765-771.

14. Gutfinger, T., y Letan, A.-"Pretreatment of soybean oil for physical refining: evaluation of some adsorbents in phospholipids removing".-J. Am. Oil Chemists' Soc. 55 (1978) 856-59.

15. Richardson, L.-"Use of bleaching clays in processing edible oil". $-J$ Am. Oil Chemists' Soc. 55 (1978) 777-780.

16. Eicke, A.-"Situation of Bleaching in the refining process of vegetable oils and animal fats".-Fette, Seifen, Anstrichmittel 87 (1985) 355 359.

17. Moser, P.-"The stability of soybean oil: effect of time and temperature on deodorization".-J. Am. Oil Chemists' Soc. 43 (1966).

18. Jaward, I.M., Kochhar, S.P. y Hudson, B.J.-"The physical refining of edible oils. 1. Effect on component fatty acids and on triglycerides".-Leb. Wiss. Techn. 16 (1983) 289-293.

19. Naward, W.W.-"Thermal and radiolytic decomposition of lipids". Chemical changes in food during processing, 79-105.-Ed by $T$. Ridcharson \& J.W. Rinley.-Westport, Connecticut, 1985.

20. Michael, W.R.-"Dimer acids structures. The thermal dimer of norma linoleate, Methyl 9-cis 12-cis Octadecadienonate".-Lipids 1 (1966) 365-368.

21. Artman, N.R., y Alexanders, J.C.-"Characterization of some heated fat components".-J. Am. Oil Chemists' Soc. 45 (1968) 643-648.

22. Sleeter, R.T.-“Effects of processing on quality of soybean oil". - J. Am. Oil. Chemists' Soc. 58 (1981) 239-247.

23. Jaward, I.M., Kochhar, S.P. y Hudson, B.J.-"The physical refining of edible oils. 2. Effects on unsaponifiable components".-Leb. Wiss. Tech. 17 (1984) 155-159.

24. Stage, H.-“The physically refining process".-J. Am. Oil. Chemists'. Soc. 62 (1985) 299-306.

25. Jaward, I.M., Kochhar, S.P. y Hudson, B.J.-"Quality characteris tics of physically refined soybean oil. Effect of pretreatment and processing time and temperature".-Food Technol. 18 (1983) 353360.

26. Wheeler, D.H. y White, J.-"Thermal reactions of Methyl Linoleate III. Characterization of $C_{18}$ cyclic esters".J. Am. Oil Chemists Soc. 44 (1967).

(Recibido: Junio 1990) 